\title{
An Unusual Presentation of Tuberculous Meningitis
}

\author{
Shahnaz Sali ${ }^{1}$; Niloufar Valipour ${ }^{1, *}$ \\ ${ }^{1}$ Infectious Diseases and Tropical Medicine Research Center Shahid Beheshti University of Medical Sciences,Tehran,IR Iran \\ ${ }^{*}$ Corresponding author: Niloufar Valipour, Infectious Disease and Tropical Medicine Research Center, Shahid Beheshti University of Medical Sciences, Tehran, IR Iran. Tel: +98- \\ 9394880188, Fax:+98-2122546026, E-mail:v_onil@yahoo.com
}

Received: December 21, 2014; Revised: May 8, 2015; Accepted: May 15, 2015

\begin{abstract}
Introduction: Tuberculosis (TB) is one of the most lethal infectious diseases, responsible for high levels of mortality and morbidity. Tuberculous meningitis (TBM) is one of the most severe presentations of extra-pulmonary tuberculosis. Due to its nonspecific signs and symptoms, diagnosis delay and neurological sequelae are common. Thus, it is important to report rare TBM manifestations.

Case Presentation: A 66-year-old diabetic man was admitted to our hospital. He complained of prolonged left otalgia and headache. Antibiotic therapy was started for mastoiditis and otitis media. Since his pain did not subside, glucocorticoid therapy was later prescribed to treat suspected temporal arteritis. However, the patient did not respond to treatment. Additional investigations such as imaging and laboratory data were performed, and the patient was finally diagnosed with TBM. The patient subsequently underwent anti-TB treatment. Conclusions: Clinicians should be aware that TBM might have unusual presentations. Early diagnosis and treatment are essential to decrease mortality and morbidity such as irreversible neurological deficits.
\end{abstract}

Keywords: Tuberculosis; Meningitis; Temporal Arteritis

\section{Introduction}

Tuberculosis (TB) is one of the most lethal infectious diseases, responsible for high levels of mortality and morbidity worldwide (1-3). TB affects one-third of the world's population (1-4), and two to three million people die from TB annually. Most of these deaths are patients in developing countries (1-5).

The clinical presentation of TB may be pulmonary or extra-pulmonary. Extra-pulmonary TB comprises approximately $15 \%$ of cases worldwide (6), and $22.5 \%$ of reported cases in Iran (7). Central nervous system (CNS) TB constitutes $5 \%$ to $15 \%$ of extra-pulmonary TB cases (1-8). Tuberculous meningitis (TBM), tuberculoma, tubercular abscess, and disseminated miliary lesions are different types of CNS-TB (3). TBM is the most severe manifestation of CNS-TB $(2,3)$ and is common in developing countries, accounting for about $16 \%$ of all of extra-pulmonary TB cases in Iran (7). The TBM mortality rate is reportedly high (about 50\%) (3, 9). The symptoms of TBM occur as primary and secondary phases. The primary symptoms include low-grade fever, loss of appetite, weight loss, headache, and gastrointestinal symptoms (e.g., vomiting). The secondary phase included reduced level of consciousness $(1,4)$. Disease complications include hydrocephalus, hemorrhage, infarction, etc. $(2,4)$. TBM diagnosis is often difficult due to its nonspecific signs and symptoms and its similarity to bacterial meningitis and other cerebrovascular events
(2). Late and unusual manifestations of TBM are common in developing countries like India and Iran (1). Late diagnosis of TBM due to nonspecific symptoms could lead to high mortality and morbidity. Therefore, due care and special attention to rare manifestations are essential. Physicians should be alert about TBM to reduce brain sequelae during early diagnosis and treatment.

\section{Case Presentation}

The patient was a 66-year-old diabetic man initially attended by a physician at our clinic. He complained of prolonged headache and otalgia and history of discharge in his left ear during the previous six months. He had no fever at the time of his visit. Based on the patient's past medical history, he had three admissions to other centers and received several antibiotics (oral: cotrimoxazole, ofloxacine and cefexim; ear drop: ciproflocin) for mastoiditis and otitis media. He had decreased hearing and the discharge from his left ear had stopped 3 months after the onset of his primary symptoms. Imbalance and headache were added to the left otalgia as of 3 month ago. He was admitted to our hospital and antibiotic therapy (intravenous ceftriaxone and otic ciprofloxacin) was started with an initial differential diagnosis of otitis media and mastoiditis. The treatment was continued for 14 days. Due to non-resolved symp-

Copyright (C) 2015, Infectious Diseases and Tropical Medicine Research Center. This is an open-access article distributed under the terms of the Creative Commons Attribution-NonCommercial 4.0 International License (http://creativecommons.org/licenses/by-nc/4.0/) which permits copy and redistribute the material just in noncommercial usages, provided the original work is properly cited. 
toms, left temporal arteritis was suspected and an earnose-throat (ENT) and rheumatology consultation was requested. The rheumatologist recommended temporal artery biopsy. However, the patient did not accept the recommended procedure. Corticosteroid therapy was therefore initiated. There was no improvement after 10 days. At this time, the patient did not complain of fever, nausea, or vomiting. However, he did complain of bilateral decreased vision. Important findings during physical examination included $6^{\text {th }}$ nerve palsy, decreased left visual quality, minor erythema on the left ear, and bilateral basal fine crackles in the lungs. Another finding included bilateral pitting edema of his lower extremities and multiple ecchymotic patches. The patient did not have meningeal irritation signs or any cardiovascular, renal, or gastrointestinal signs and symptoms.

Laboratory findings included the following levels and concentrations: white blood cell $(\mathrm{WBC})=21000 / \mu \mathrm{L}, 97 \%$ neutrophil, blood urea nitrogen $(\mathrm{BUN})=77 \mathrm{mg} / \mathrm{dl}$, creatinine $(\mathrm{Cr})=1.83 \mathrm{mg} / \mathrm{dl}$, Hemoglobin $(\mathrm{Hb})=10.2 \mathrm{~g} / \mathrm{dl}$, platelet $(\mathrm{PLT})=303,000 / \mu \mathrm{L}$, fasting blood sugar $(\mathrm{FBS})=$ $271 \mathrm{mg} / \mathrm{dl}$, sodium $(\mathrm{Na})=129 \mathrm{mEq} / \mathrm{L}$, potassium $(\mathrm{K})=4.6$ $\mathrm{mEq} / \mathrm{L}$, erythrocyte sedimentation rate $(\mathrm{ESR})=48 \mathrm{~mm} / \mathrm{h}$, C-reactive protein $(\mathrm{CRP})=3+$, and liver function test $(\mathrm{LFT})$ $=$ normal range.

Left side mastoiditis was visible in brain computer tomog raphy (CT) (Figure 1). There were no findings suggestive of pupilitis during ophthalmology consultation. A CT scan was recommended before lumbar puncture (LP). Brain shifting and other contraindications of LP were not seen. LP was therefore performed to test for chronic meningitis.

Based on these findings, the patient was diagnosed with

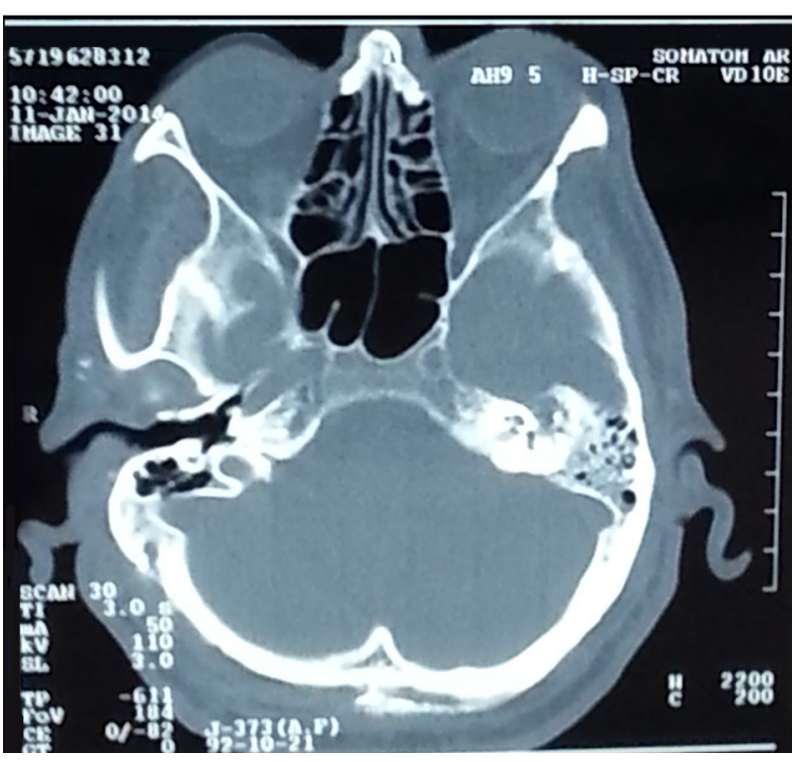

Figure 1. Brain CT (Mastoiditis) meningitis and started on intravenous meropenem and vancomycin. All laboratory tests to rule out other conditions besides chronic meningitis, such as sarcoid Analysis of the lumbar puncture sample revealed: $\mathrm{WBC}=170$ $\mathrm{mm}^{3}$, lymphocyte $=90 \%$, neutrophil $=10 \%$, red blood cell $(\mathrm{RBC})=50 \mathrm{~mm}^{3}$, glucose $=59 \mathrm{mg} / \mathrm{dl}$, blood sugar $=$ $144 \mathrm{mg} / \mathrm{dl}$, protein $=39 \mathrm{mg} / \mathrm{dl}$, and adenosine deaminase $(\mathrm{ADA})=19 \mathrm{U} / \mathrm{L}$.

osis, brucellosis, syphilis, fungi, and tuberculosis, were requested. Wright, 2-mercaptoethanol (2ME), venereal disease research laboratory (VDRL), and rheumatoid factor test results were normal. However, the patient tested positive for TB by polymerase chain reaction (PCR) after 2 days. His sputum smear was negative for mycobacterium and other organisms, and the induration of the purified protein derivative (PPD) test was $3 \mathrm{~mm}$. The patient's chest x-ray was normal. He was also negative for human immunodeficiency virus (HIV).

Because the patient did not respond to previous treatments and his cerebrospinal fluid (CSF) tested positive for TB by PCR, the temporal arteritis diagnosis was not a priority. At this stage, TBM appeared to be the most likely diagnosis. Hydrocortisone and a quadruple TB drug treatment (isoniazid, ethambutol, pyrazinamide, and rifampin) at standard doses were initiated. However, the patient's Glasgow coma scale (GCS) score dropped to 3/15 within 2 days. Magnetic resonance imaging (MRI) performed due to his low GCS score revealed cerebrovascular accidents (CVAs) in his cerebellum and occipital lobe (Figure 2), possibly caused by TB vasculitis. The patient died 2 weeks later. Two months after starting the CSF culture, the results were negative.

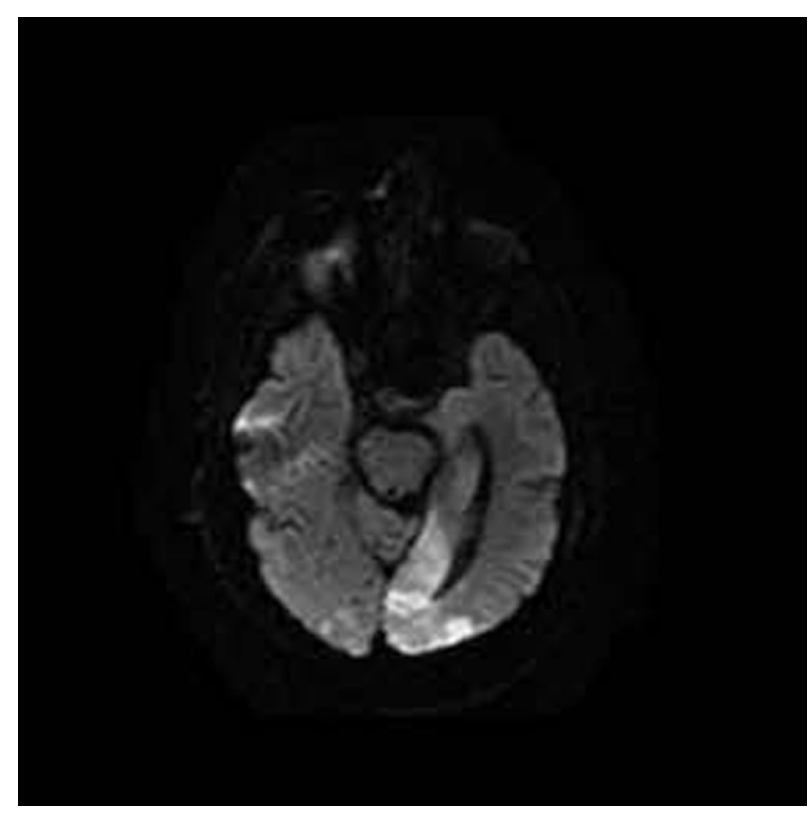

Figure 2. Brain MRI Showing Cerebrovascular Accident in the Cerebellum and Occipital Lobe 


\section{Discussion}

While primary TB infection involves the lung, extrapulmonary involvement is also possible. Lymph node TB is the most common manifestation of extra-pulmonary $\mathrm{TB}$, followed by involvement of the pleura, genitourinary system, and bones and joints. Peritoneal, pericardial, and meningeal TB are uncommon presentations of extra-pulmonary TB (10). Although TB is rare in developed countries, its high prevalence in developing nations causes many problems (11). Late diagnosis is a common cause of death TB patients (50\% of cases). TBM begins as a primary infection of the lungs or rarely following consumption of infected milk (1). The clinical manifestations of TBM include hydrocephalus, hemorrhage, infarction $(2,4)$, cranial nerve palsies, inflammation of brain parenchyma, alteration of sensorium, focal neurological lesions, and brain edema (1). TBM manifestations may also be constitutional and nonspecific, leading to delays in diagnosis and subsequent increased mortality and morbidity, including neurological deficits. Therefore, it is important to report rare TBM manifestations. TBM symptoms occur in primary and secondary phases. The primary symptoms include low-grade fever, loss of appetite, weight loss, headache, and gastrointestinal symptoms (e.g., vomiting). The secondary phase includes decreased the level of consciousness $(1,4)$. In a review article of 20 TBM cases, fever was the most common manifestation, occurring in $75 \%$ of cases. Other presentations included headache (about 40\%), for motor deficit (40\%), and convulsions (15\%) (12). In other studies from industrialized countries, the prevalence of CNS-TB was high in women and immigrants people, suggesting a significant relationship between gender and ethnicity in TB infection (13, 14). Immune status is very important in patients infected with TB; therefore, these patients should also be screened for HIV, diabetes mellitus, and vitamin D deficiency (15). A review article of 50 TBM cases reported 19\% mortality, $48 \%$ morbidity (e.g. neurological deficit), and a 33\% cure rate. Therefore, early diagnosis and treatment are essential (2). The primary presentation of TBM in our patient was prolonged otalgia and headache that led to altered consciousness. After ruling out other etiologies based on laboratory data, imaging, and PCR findings, TBM was the only remaining diagnosis. Rarely, the source of TBM is an adjacent extraneural infection in the ear, vertebrae, or mastoid sinuses (1). Osteomyelitis in TB is also rare (11), but our patient had osteomyelitis of the mastoid bone. In recent studies, most patients were young (mean 21 years) $(1,4,9)$, but the patient in our study was 66 years old. Without biopsy specimens, our diagnosis was based on imaging, CSF analysis, serology, and treatment response (12). Overall, CSF analysis of these patients typically shows high leukocyte count (usually lymphocyte dominant), highly protein content, and low glucose concentration $(1,2,9,16)$. PCR testing has a sensitivity of up to $80 \%$, but a false positive rate around $10 \%$ (1). A previous study re- ported TBM diagnosis sensitivity and specificity of $38 \%$ and $100 \%, 89 \%$ and $90 \%$, and $6 \%$ and $100 \%$ for CSF culture, TB-PCR, and sputum smear, respectively (17). In another case report of a 4 year old child, PPD test, TB-PCR, and CSF culture were negative for TB; however, biopsy of a mass lesion in the spinal cord showed chronic granulomatosis that proved to be TB-associated pachymeningitis (18). CSF $\mathrm{ADA}>8.0 \mathrm{U} / \mathrm{L}$ has a sensitivity and specificity of $80 \%$ and $90 \%$, making it a useful marker for diagnosis of TBM (19). TBM treatment requires administration of drugs able to cross the blood brain barrier that are also effective in the CNS. In our study, we used first-line drugs for treatment of TB (isoniazid, rifampin, ethambutol, and pyrazinamide). Second-line drugs may be used in cases of resistance to first-line drugs. An Egyptian study reported low resistance to first-line drugs (20). The treatment duration is about 9 to 12 months. Administration of intravenous glucocorticoids decreases intracranial pressure, especially in obstructive hydrocephalus. Intravenous glucocorticoids facilitated the response to treatment (1).

TB is one of the most common infectious diseases, and TBM is the most severe presentation of extra pulmonary TB. Because distinguishing between symptoms of TBM and other etiologies of bacterial meningitis or cerebral events is challenging, late diagnosis and treatment are common, which contributes to high mortality rates and irreversible cerebral lesions. Thus, physicians should be aware of rare TBM manifestations. Although TBM is very rare and osteomyelitis in TBM is extraordinarily so, it must be considered as a differential diagnosis in patients with otalgia, headache, and mastoiditis.

\section{Acknowledgements}

The authors thank Labbafi Nejad Teaching and Medical Hospital for its support in carrying out this study.

\section{Authors' Contributions}

Data collection and analysis: Niloufar Valipour. Manuscript drafting and writing: Shahnaz Sali and Niloufar Valipour. Critical manuscript revision: Shahnaz Sali. All authors have read and approved the paper.

\section{References}

1. Shankaragouda BH, Barjatya $\mathrm{H}$, sahu U, Savadkar A. A case of tuberculous meningitis presenting with cognitive defects. Int $\mathrm{J} \mathrm{Nu}$ trition Pharmacol Neurol Dis. 2013;3(4):388-91.

2. Christensen AS, Andersen AB, Thomsen VO, Andersen PH, Johansen IS. Tuberculous meningitis in Denmark: a review of 50 cases. BMC Infect Dis. 2011;11:47.

3. Garg K, Gurjar HK, Joseph SG, Shashikant RC, Sharma BS. Third ventricular tuberculoma. Neurol India. 2014;62(1):73-5.

4. Komolafe MA, Sunmonu TA, Esan OA. Tuberculous meningitis presenting with unusual clinical features in Nigerians: Two case reports. Cases J. 2008;1(1):180.

5. Chaudhary S, Kalra N, Gomber S. Tuberculous osteomyelitis of the mandible: a case report in a 4-year-old child. Oral Surg Oral Med Oral Pathol Oral Radiol Endod. 2004;97(5):603-6.

6. Andreoli TE. Cecil Essential of Medicine. 8 edPhiladelphia: Saunders-Elsevier; 2010. 
7. Hatami H, Azizi F. Tuberculosis epidemiology and controlling common diseases in Iran.Tehran: Eshtiagh; 2001.

8. Vajramani GV, Devi BI, Hegde T, Santosh V, Khanna N, Vasudev MK. Intraventricular tuberculous abscess: a case report. Neurol India. 1999;47(4):327-9.

9. Ranganathan N, Hogarth K. Tuberculous meningitis. BMJ Case Rep. 2013;2013.

10. Fauci AS, Braunwald E, Kasper DL. Harrison's principles of internal medicine.New York: McGraw-Hill; 2005.

11. Natarajarathinam G, Rao A, Palanimuthu S, Kannaperuman J. Primary tuberculous osteomyelitis of the mandible: A rare case report. Dent Res J. 2013;10(2):283-6.

12. Daniele B. Characteristics of central nervous system tuberculosis in a low-incidence country: a series of 20 cases and a review of the literature. Jpn J Infect Dis. 2014;67(1):50-3.

13. Forssbohm M, Zwahlen M, Loddenkemper R, Rieder HL. Demographic characteristics of patients with extrapulmonary tuberculosis in Germany. Eur Respir J. 2008;31(1):99-105.

14. Lillebaek T, Andersen AB, Dirksen A, Smith E, Skovgaard LT, KokJensen A. Persistent high incidence of tuberculosis in immigrants in a low-incidence country. Emerg Infect Dis. 2002;8(7):679-84.
15. Afrozul H. Global vitamin D deficiency and its role in health and disease. Nt J Nutr Pharmac Neurol Dis. 2013;3(58):288-91.

16. Porkert MT, Sotir M, Parrott-Moore P, Blumberg HM. Tuberculous meningitis at a large inner-city medical center. Am J Med Sci. 1997;313(6):325-31.

17. Naserpour Farivar T, P. J. , Hashemi Shahri M, Naderi M, Alavi R, Batoul S. Mood Sensitivity and Specificity of TaqMan Real Time PCR, PCR, Microscopy and Culture in Diagnosis of Tuberculous Meningitis in a High Incidence of Tuberculosis Province in Southeast of Iran. Biotech Health Scis. 2014;1(1):e19194.

18. Karimi A, Rafiei Tabatabaei S, Shiva F, Sayfikar M, Jafari M, Shirvani F, et al. Tuberculous Pachymeningitis in a Young Child With Spinal Involvement: A Case Report. Arch Pediatr Infect Dis. 2013;1(1):36-9.

19. Sakhelashvili MI, Stadovich NM, Omelian OV, Nakonechnyi ZM [Pathology of tuberculosis meningitis in adults (autopsy findings)]. Lik Sprava. 2001(3):33-6.

20. Girgis NI, Sultan Y, Farid Z, Mansour MM, Erian MW, Hanna LS, et al. Tuberculosis meningitis, Abbassia Fever Hospital-Naval Medical Research Unit No. 3-Cairo, Egypt, from 1976 to 1996. Am J Trop Med Hyg. 1998;58(1):28-34. 